SUSTAINABLE FORESTRY

COLLECTION 65-66, 2012
ODRŽIVO ŠUMARSTVO

ZBORNIK RADOVA 65-66, 2012

UDK $630 * 181.45+630 * 425(497.11)(23.03$ Kopaonik $)=111$

Original scientific paper

\title{
INTENSIVE MONITORING AT LEVEL II TEST PLOT KOPAONIK IN 2012
}

\author{
Svetlana BILIBAJKIĆ, Tomislav STEFANOVIĆ, Radovan NEVENIĆ, \\ Zoran PODUŠKA, Renata GAGIĆ SERDAR, Ilija ĐORĐEVIĆ, \\ Goran ČEŠLJAR, Zoran MILETIĆ ${ }^{1}$
}

\begin{abstract}
Level II intensive monitoring of forest vitality represents a multipurpose research system. Forest ecosystems are highly complex entities characterized by numerous different parameters subject to continuous variation due to constant and mutually inseparable effects of both biotic and abiotic factors. Evaluation criteria applied in intensive monitoring are compatible and defined in such a manner that, subsequent to their recording and statistical processing, data obtained on the condition of forests are easy to compare both analytically and logically, thus providing the basis for a variety of comparative studies. Dedicated test plot for intensive monitoring of trans-boundary air pollution impact on forest ecosystems in Serbia, a Level II test plot, was established in Kopaonik in 2010, with ten panels - from 10 separate forestry research areas, grouped according to the research subjects, which methodology is prescribed by ICP Forests Manual. This paper presents the results of intensive monitoring of parameters under review at Level II test plot Kopaonik in 2012.
\end{abstract}

Key words: Level II test plot Kopaonik, intensive monitoring, crown condition, defoliation, deposition, litterfall.

\section{INTENZIVNI MONITORING NA OGLEDNOM POLJU NIVO-a II „KOPAONIK“ U 2012.GODINI}

\begin{abstract}
Intenzivni monitoring vitalnosti šuma Nivo-a II, predstavlja višenamenski sistem predmetnih istraživanja. Šumski ekosistem, kao izuzetno složen entitet, odlikuju različiti parametri podložni konstatnim varijacijama usled neprestanog $i$
\end{abstract}

\footnotetext{
${ }^{1}$ Institute of Forestry, Belgrade, Kneza Viseslava No 3, Serbia
} 
neodvojivog delovanja abiotičkih $i$ biotičkih činilaca. Kriterijumi procene koje intenzivni monitoring podrazumeva, usaglašeni su i tako određeni da se dobijeni podaci o stanju šuma, nakon unosa i statističke obrade analitički i logički lako porede, dajući osnovu za različite komparativne studije. Namenska ogledna površina za intenzivni monitoring uticaja prekograničnog vazdušnog zagađenja na šumske ekosisteme u Srbiji - bioindikacijska tačka Nivo-a II osnovana je u 2010. godini na Kopaoniku, sa deset radnih panela - iz 10 zasebnih stručnih oblasti šumarstva, grupisanih prema predmetu istraživanja, a metodološki propisanim Manual-om ICP-a za šume. U radu su dati rezultati intenzivnog monotoringa praćenih parametara u 2012.godini na BIT Nivo-a II na Kopaoniku

Ključne reči: BIT Nivo II Kopaonik, intenzivni monitoring, stanje krošnji, defolijacija, depozicija, lisni opad.

\section{INTRODUCTION}

Level II monitoring of forest vitality is a versatile system of comparative research of many different forestry disciplines. Scientific research in forest condition monitoring is characterized by a multi-disciplinary and studious approach. Level II monitoring test stations have been installed all over the European continent according to the uniform ICP Forests methodology in order to enable continuous measurement and collection of data on condition of forests with various specific environmental circumstances. Such forest biocenoses belong to different taxonomic groups with a wide range of differences in species diversity and extent of man's impact in terms of intensifying their productive function; there are also forests where explicit management mechanisms of habitat maintenance are applied with strict protection and conservation regimes in effect. The objective of such research approach is to enable analyses performed over several years to allow observing patterns and drawing conclusions on the phenomenon of forest drying in Europe as well as clearer defining the cause-effect system for all changes monitored. Evaluation criteria applied in intensive monitoring are compatible and defined in such a manner that, subsequent to their recording and statistical processing, data obtained on the condition of forests are easy to compare both analytically and logically, thus providing the basis for a variety of comparative studies. By perceiving similarities and dissimilarities, assumptions on the primary causes of the disturbed natural equilibrium in the forest biocenoses are rejected or accepted, further progress of the changes is anticipated and further degradation of forests as invaluable natural entities is prevented strategically, from the aspects of multiple applied forestry disciplines.

By setting up test stations in Kopaonik National Park (2010), Fruška Gora (2009) and Odžaci (2011), Serbia joined the European network of over 800 Level II test plots.

\section{MATERIALS AND METHODS}

A Level II test plot, a dedicated test plot for intensive monitoring of transboundary air pollution impact on forest ecosystems in Serbia, was established in Kopaonik in 2010. The test plot is situated in the 74th division of the estate 
"Samokovska reka" in Kopaonik National Park, within pure spruce stand, Picea abies (L.) H.Karst., which forms thick forest complexes in such altitude $(1,720 \mathrm{~m})$. Level II sample plot covers an area of 0.5 ha $(100 \times 50 \mathrm{~m})$. Within its area there are three subplots for customized sampling and a buffer zone. The trees are marked with permanent bark markings, i.e. numbers from 1 to 195 . Current status situation plan $^{1}$ of the test plot is provided in Figure 1.
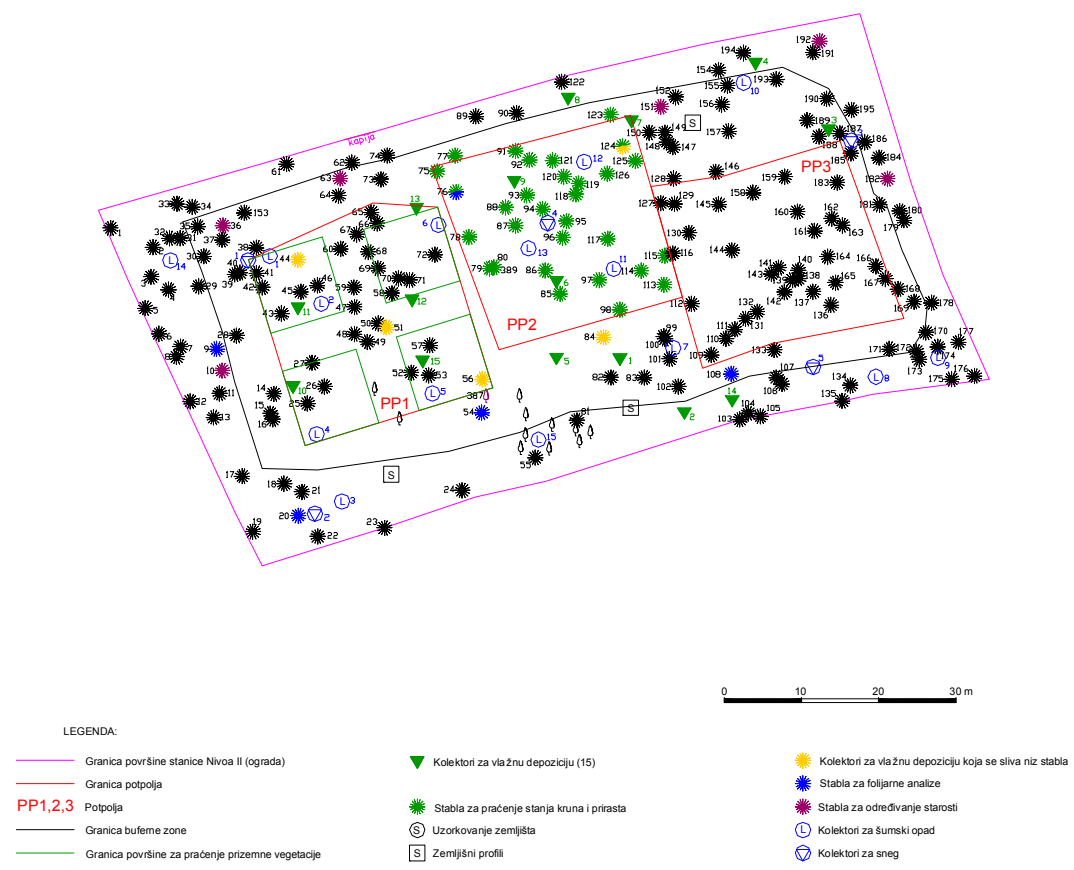

Figure 1. Current status situation plan of the test plot area

Level II monitoring program comprised the following parameter groups: crown condition, foliar analyses, soil chemistry, soil solution chemistry, growth and yield, ground vegetation, atmospheric deposition, air quality, meteorology, phenology and forest litterfall. Frequency of monitoring individual parameters is shown in Table 1.

Table 1. Parameters, frequency and intensity of Level II monitoring

\begin{tabular}{|l|l|}
\hline \multicolumn{1}{|c|}{ Parameter type } & \multicolumn{1}{c|}{ Frequency of monitoring } \\
\hline Crown condition & At least annually \\
\hline Foliar analyses & Every two years \\
\hline Soil chemistry & Every ten years \\
\hline Soil solution chemistry & Continuously \\
\hline Growth and yield & Every five years \\
\hline Ground vegetation & Every five years \\
\hline Atmospheric deposition & Continuously \\
\hline Air quality & Continuously \\
\hline
\end{tabular}

\footnotetext{
${ }^{1}$ Current status situation plan was prepared at the Forestry Institute in Belgrade, in a digital form, according to the field status and the blueprint of the basic layout of the test subplots drawn by the team of the Faculty of Forestry in Belgrade in 2010.
} 


\begin{tabular}{|l|l|}
\hline \multicolumn{1}{|c|}{ Parameter type } & \multicolumn{1}{c|}{ Frequency of monitoring } \\
\hline Ozone injuries & Annually \\
\hline Meteorology & Continuously \\
\hline Phenology & Several times a year \\
\hline Forest litterfall & Continuously \\
\hline
\end{tabular}

Assessment of the crown condition in Level II intensive monitoring encompasses assessment of defoliation, injury detection, tree condition, crown shade (damage), crown visibility, fructiferousness of the visible crown parts and presence of the secondary sprouts. Out of the total number of spruce trees marked, 30 trees were selected within subplot 2 for the purpose of crown condition monitoring.

Phenological observations included 15 selected spruce trees (Picea abies L.). Phenophases were observed continuously, in succession, and the following parameters were detected and monitored: budding, change in color of conifer needles, significant indications of needle or crown damage, other injuries (broken branches and trees and uprooted trees), secondary budding and blooming. The aforesaid parameters were monitored in the trees located within the test plot, starting from the first field visit.

For litterfall sampling 15 collector pads for collection of dead organic remnants of forest trees (litterfall). The average collection surface area was 500 $\mathrm{cm}^{2}$ per pad and the total collection surface area amounted to $0.75 \mathrm{~m}^{2}$.

For wet deposition collection within the test plot, 15 collectors of precipitation falling through the tree crowns ("throughfall"), 5 collectors of deposition sliding down the tree trunks ("steamflow") and 5 snow collectors ("bulk") were placed in appropriate positions.

Soil solution is sampled by means of gravitational lysimeters placed into the front vertical wall of the existing pedological profiles at defined depths below the organic layer horizon.

For meteorological monitoring performed in order to obtain information on microclimatic conditions, data provided by Kopaonik automatic weather station of the Hydrometeorological Service of Serbia, which is situated near the Level II test plot on Mt. Kopaonik, were used. The location of the weather station ensured representative meteorological data according to the ICP Forests Manual. The following mandatory parameters were monitored: precipitation (PR), air temperature (AT), relative air humidity (RH), wind speed (WS), wind direction (WD) and solar radiation (SR).

At the end o 2011, the measuring instruments were checked and prepared for the climatic conditions typical of long and severe winters at such altitudes.

In April 2012, the equipment was washed and sterilized and minimum required repairs were performed. Phenological observations were conducted continuously. Wet deposition collection and soil solution sampling were carried out on a monthly basis. Upon each field visit, litterfall collectors were emptied.

\section{RESULTS AND DISCUSSION}

During 2012 parameters with continuous and an annual-basis frequency of monitoring were monitored. 


\subsection{Assessment of the Crown Condition - 2012 Intensive Monitoring}

Crown condition assessment of the trees at the Level II test plot Kopaonik was performed as at August 23, 2012. As in prior years, the assessment included 30 spruce trees selected for annual crown condition monitoring in subplot 2 .

Table 2. XX2012. (PLT) Data on the subplot dedicated for crown condition assessment, Level II, Kopaonik

\begin{tabular}{|c|c|c|c|c|c|c|c|c|}
\hline No. & State code & Subplot no. & $\begin{array}{c}\text { Assessment } \\
\text { date }\end{array}$ & Latitude & Longitude & $\begin{array}{c}\text { Altitude } \\
\text { code }\end{array}$ & $\begin{array}{c}\text { Team } \\
\text { identification }\end{array}$ & $\begin{array}{c}\text { Other } \\
\text { findings }\end{array}$ \\
\hline 1 & 67 & 2 & 230812 & $+43^{0} 17^{\prime} 30^{\prime \prime}$ & $+20^{0} 48^{\prime} 50^{\prime \prime}$ & 35 & REIGO & \\
\hline
\end{tabular}

Crown condition assessment focused on determining the degree of defoliation, tree drying and removal, tree status, crown shade, crown visibility, foliage transparency and other findings.

Injuries were also detected in selected trees. For each tree where injury/damage was identified, location, symptom, cause and intensity of injury/damage are stated.

Table 3. XX2012. (TRC) Crown condition parameters, Level II Kopaonik

\begin{tabular}{|c|c|c|c|c|c|c|c|c|c|c|c|}
\hline No. & $\begin{array}{c}\text { Sub- } \\
\text { plot no. }\end{array}$ & $\begin{array}{c}\text { Assessment } \\
\text { date }\end{array}$ & Tree no. & Species & $\begin{array}{l}\text { Drying - } \\
\text { removal }\end{array}$ & $\begin{array}{l}\text { Tree } \\
\text { status }\end{array}$ & $\begin{array}{l}\text { Crown } \\
\text { shade }\end{array}$ & $\begin{array}{l}\text { Crown } \\
\text { visibility }\end{array}$ & Defoliation & $\begin{array}{c}\text { Foliage } \\
\text { transparency }\end{array}$ & $\begin{array}{l}\text { Other } \\
\text { findings }\end{array}$ \\
\hline 1 & 2 & 230812 & 75 & 118 & 01 & 1 & 2 & 2 & 10 & 25 & U.b. * \\
\hline 2 & 2 & 230812 & 76 & 118 & 01 & 1 & 1 & 2 & 10 & 20 & U.b. ${ }^{*}$ \\
\hline 3 & 2 & 230812 & 78 & 118 & 01 & 1 & 2 & 2 & 10 & 25 & U.b. ${ }^{*}$ \\
\hline 4 & 2 & 230812 & 79 & 118 & 01 & 1 & 1 & 2 & 15 & 25 & U.b. ${ }^{*}$ \\
\hline 5 & 2 & 230812 & 80 & 118 & 01 & 1 & 1 & 2 & 20 & 25 & U.b. ${ }^{*}$ \\
\hline 6 & 2 & 230812 & 85 & 118 & 01 & 1 & 2 & 2 & 15 & 25 & U.b. ${ }^{*}$ \\
\hline 7 & 2 & 230812 & 86 & 118 & 01 & 1 & 3 & 3 & 15 & 30 & U.b. ${ }^{*}$ \\
\hline 8 & 2 & 230812 & 87 & 118 & 01 & 3 & 3 & 3 & 30 & 70 & U.b. ${ }^{*}$ \\
\hline 9 & 2 & 230812 & 88 & 118 & 38 & 5 & 6 & 2 & 100 & 99 & U.b. ${ }^{*}$ \\
\hline 10 & 2 & 230812 & 91 & 118 & 41 & & & & & & Felled \\
\hline 11 & 2 & 230812 & 92 & 118 & 01 & 2 & 3 & 3 & 30 & 60 & U.b. ${ }^{*}$ \\
\hline 12 & 2 & 230812 & 93 & 118 & 01 & 1 & 3 & 3 & 30 & 60 & U.b. ${ }^{*}$ \\
\hline 13 & 2 & 230812 & 94 & 118 & 01 & 3 & 3 & 3 & 50 & 80 & U.b. ${ }^{*}$ \\
\hline 14 & 2 & 230812 & 95 & 118 & 01 & 2 & 3 & 3 & 20 & 30 & U.b. ${ }^{*}$ \\
\hline 15 & 2 & 230812 & 96 & 118 & 01 & 1 & 4 & 4 & 20 & 30 & U.b. * \\
\hline 16 & 2 & 230812 & 97 & 118 & 01 & 1 & 3 & 3 & 20 & 50 & U.b. ${ }^{*}$ \\
\hline 17 & 2 & 230812 & 98 & 118 & 01 & 1 & 3 & 3 & 20 & 80 & U.b. ${ }^{*}$ \\
\hline 18 & 2 & 230812 & 113 & 118 & 01 & 1 & 2 & 2 & 25 & 60 & U.b. * \\
\hline 19 & 2 & 230812 & 114 & 118 & 01 & 1 & 4 & 3 & 25 & 80 & $U . b .^{*}$ \\
\hline 20 & 2 & 230812 & 115 & 118 & 01 & 1 & 3 & 3 & 30 & 50 & U.b. ${ }^{*}$ \\
\hline 21 & 2 & 230812 & 117 & 118 & 01 & 1 & 4 & 3 & 25 & 50 & U.b. ${ }^{*}$ \\
\hline 22 & 2 & 230812 & 118 & 118 & 01 & 1 & 3 & 2 & 20 & 65 & U.b. ${ }^{*}$ \\
\hline 23 & 2 & 230812 & 119 & 118 & 01 & 3 & 3 & 3 & 95 & 90 & U.b. ${ }^{*}$ \\
\hline 24 & 2 & 230812 & 120 & 118 & 01 & 1 & 1 & 2 & 30 & 40 & U.b. ${ }^{*}$ \\
\hline 25 & 2 & 230812 & 121 & 118 & 01 & 1 & 3 & 3 & 15 & 20 & U.b. * \\
\hline 26 & 2 & 230812 & 124 & 118 & 01 & 1 & 2 & 2 & 25 & 60 & U.b. ${ }^{*}$ \\
\hline
\end{tabular}




\begin{tabular}{|c|c|c|c|c|c|c|c|c|c|c|c|}
\hline No. & $\begin{array}{c}\text { Sub- } \\
\text { plot no. }\end{array}$ & $\begin{array}{c}\text { Assessment } \\
\text { date }\end{array}$ & Tree no. & Species & $\begin{array}{c}\text { Drying - } \\
\text { removal }\end{array}$ & $\begin{array}{c}\text { Tree } \\
\text { status }\end{array}$ & $\begin{array}{c}\text { Crown } \\
\text { shade }\end{array}$ & $\begin{array}{c}\text { Crown } \\
\text { visibility }\end{array}$ & Defoliation & $\begin{array}{c}\text { Foliage } \\
\text { transparency }\end{array}$ & $\begin{array}{c}\text { Other } \\
\text { findings }\end{array}$ \\
\hline 27 & 2 & 230812 & 125 & 118 & 38 & 5 & 3 & 3 & 100 & 99 & U.b. ${ }^{*}$ \\
\hline 28 & 2 & 230812 & 126 & 118 & 01 & 1 & 2 & 2 & 15 & 40 & U.b. ${ }^{*}$ \\
\hline 29 & 2 & 230812 & 77 & 118 & 01 & 1 & 3 & 2 & 15 & 30 & U.b..$^{*}$ \\
\hline 30 & 2 & 230812 & 123 & 118 & 01 & 1 & 1 & 1 & 15 & 20 & U.b..$^{*}$ \\
\hline
\end{tabular}

*Usnea barbata

Tables 3 and 4 present crown condition parameters and injury/damage parameters at the Level II test plot Kopaonik.

Table 4. XX2012. (TRD) Injury/Damage parameters, Level II Kopaonik

\begin{tabular}{|c|c|c|c|c|c|c|c|c|c|c|c|c|}
\hline No. & $\begin{array}{c}\text { Sub- } \\
\text { plot no. }\end{array}$ & $\begin{array}{c}\text { Assessment } \\
\text { date }\end{array}$ & $\begin{array}{l}\text { Tree } \\
\text { no. }\end{array}$ & $\begin{array}{c}\text { Injured } \\
\text { tree part }\end{array}$ & Symptom & $\begin{array}{c}\text { Symptom } \\
\text { designation }\end{array}$ & $\begin{array}{c}\text { Crown } \\
\text { part }\end{array}$ & \begin{tabular}{|c|} 
Time of \\
injury/damage \\
inception
\end{tabular} & Cause & $\begin{array}{c}\text { Cause } \\
\text { description }\end{array}$ & $\begin{array}{l}\text { Injury/ } \\
\text { Damage } \\
\text { intensity }\end{array}$ & $\begin{array}{l}\text { Other } \\
\text { findings }\end{array}$ \\
\hline 1 & 2 & 230812 & 75 & 00 & & & & & & & & U.b. ${ }^{*}$ \\
\hline 2 & 2 & 230812 & 76 & 00 & & & & & & & & U.b. ${ }^{*}$ \\
\hline 3 & 2 & 230812 & 78 & 11 & 02 & 38 & 3 & 3 & 301 & CHRYABI & 1 & U.b. ${ }^{*}$ \\
\hline 4 & 2 & 230812 & 79 & 11 & 02 & 38 & 3 & 2 & 301 & CHRYABI & 2 & U.b. ${ }^{*}$ \\
\hline 5 & 2 & 230812 & 80 & 11 & 02 & 38 & 3 & 3 & 301 & CHRYABI & 2 & U.b. ${ }^{*}$ \\
\hline 6 & 2 & 230812 & 85 & 11 & 02 & 38 & 3 & 2 & 301 & CHRYABI & 2 & U.b. ${ }^{*}$ \\
\hline 7 & 2 & 230812 & 86 & 13 & 02 & 38 & & 3 & 999 & & 1 & U.b. * \\
\hline 8 & 2 & 230812 & 87 & 31 & 10 & 65 & & 3 & 220 & IPSTYPO & 3 & U.b. ${ }^{*}$ \\
\hline 9 & 2 & 230812 & 88 & 04 & 10 & 65 & & 3 & 220 & IPSTYPO & 7 & U.b. ${ }^{*}$ \\
\hline 10 & 2 & 230812 & 91 & 04 & 22 & & & 1 & 400 & & 7 & \\
\hline 11 & 2 & 230812 & 92 & 33 & 17 & 58 & & 2 & 500 & & 2 & $U . b{ }^{*}$ \\
\hline 12 & 2 & 230812 & 93 & 33 & 17 & 58 & & 3 & 500 & & 1 & U.b. ${ }^{*}$ \\
\hline 13 & 2 & 230812 & 94 & 33 & 17 & 58 & & 3 & 500 & & 3 & U.b. ${ }^{*}$ \\
\hline 14 & 2 & 230812 & 95 & 11 & 02 & 38 & 3 & 2 & 301 & CHRYABI & 1 & U.b. ${ }^{*}$ \\
\hline 15 & 2 & 230812 & 96 & 00 & & & & & & & & U.b. ${ }^{*}$ \\
\hline 16 & 2 & 230812 & 97 & 00 & & & & & & & & U.b. ${ }^{*}$ \\
\hline 17 & 2 & 230812 & 98 & 32 & 17 & 60 & & 3 & 999 & & 1 & U.b. ${ }^{*}$ \\
\hline 18 & 2 & 230812 & 113 & 00 & & & & & & & & U.b. ${ }^{*}$ \\
\hline 19 & 2 & 230812 & 114 & 11 & 02 & 38 & 3 & 3 & 301 & CHRYABI & 1 & U.b. ${ }^{*}$ \\
\hline 20 & 2 & 230812 & 115 & 00 & & & & & & & & U.b. ${ }^{*}$ \\
\hline 21 & 2 & 230812 & 117 & 00 & & & & & & & & U.b. * \\
\hline 22 & 2 & 230812 & 118 & 00 & & & & & & & & U.b. ${ }^{*}$ \\
\hline 23 & 2 & 230812 & 119 & 34 & 11 & 57 & & 3 & 304 & TRAMSPP & 6 & U.b. ${ }^{*}$ \\
\hline 24 & 2 & 230812 & 120 & 11 & 02 & 38 & 3 & 3 & 301 & CHRYABI & 1 & U.b. ${ }^{*}$ \\
\hline 25 & 2 & 230812 & 121 & 11 & 02 & 38 & 3 & 3 & 301 & CHRYABI & 1 & U.b. ${ }^{*}$ \\
\hline 26 & 2 & 230812 & 124 & 24 & 13 & & 2 & 2 & 999 & & 2 & U.b. ${ }^{*}$ \\
\hline 27 & 2 & 230812 & 125 & 04 & 10 & 65 & & 3 & 220 & IPSTYPO & 7 & U.b. ${ }^{*}$ \\
\hline 28 & 2 & 230812 & 126 & 00 & & & & & & & & U.b. ${ }^{*}$ \\
\hline 29 & 2 & 230812 & 77 & 00 & & & & & & & & U.b. ${ }^{*}$ \\
\hline 30 & 2 & 230812 & 123 & 00 & & & & & & & & U.b. * \\
\hline
\end{tabular}

*Usnea barbata

As compared to the previous two years, the number of trees not subject to defoliation has increased, as well as the percentage of trees with weak defoliation, whereas the percentage of trees with moderate defoliation has declined. The 
percentage of trees with strong defoliation has remained almost the same as in the previous years.

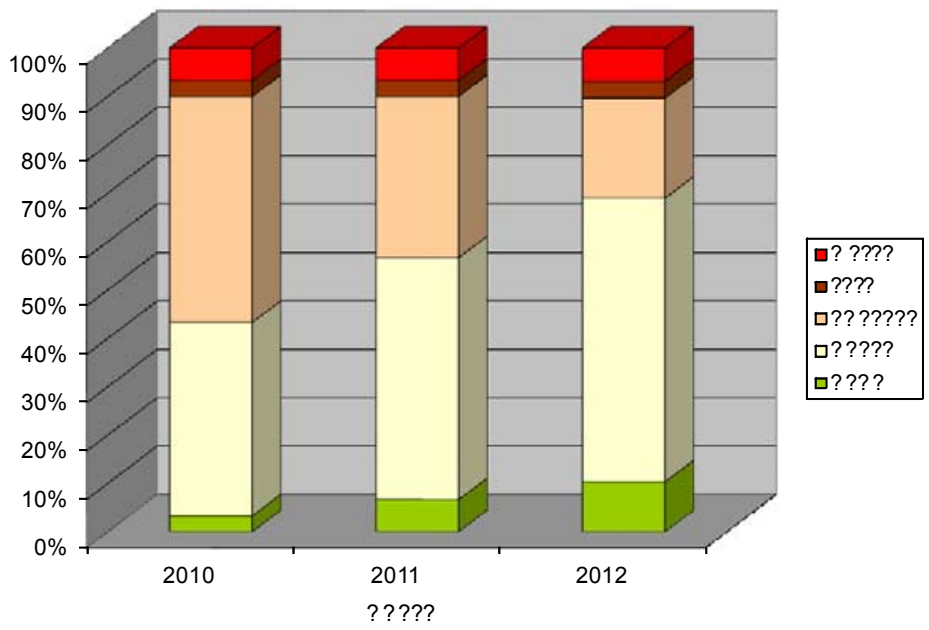

Graph 1. Comparative presentation of defoliation in the period from 10/10-10/12 - Level II, Kopaonik

\subsection{Phenological Observations in 2012}

At Level II test plot 15 spruce trees (Picea abies L.) were selected for phenological observations (Table 5).

The vegetation of the dominant species commenced rather late. Observed from different expositions of the evaluated trees, budding started in the third week of June 2012, whereafter slightly opened male and female reproductive organs were identified, which in conifer trees are called "blossoms." Pollination lasted until mid-July, which is easily perceived in mature spruce trees in the form of pollen clouds carried by the wind.

Table 5. XX 2012. (PLP) Registration of trees selected for intensive phonological monitoring

\begin{tabular}{|c|c|c|c|c|c|c|c|c|}
\hline No. & $\begin{array}{c}\text { Sub-plot } \\
\text { no. }\end{array}$ & $\begin{array}{c}\text { Species } \\
\text { code }\end{array}$ & $\begin{array}{l}\text { Placement } \\
\text { date }\end{array}$ & $\begin{array}{c}\text { Tree } \\
\text { no. }\end{array}$ & $\begin{array}{c}\text { Visible } \\
\text { crown part }\end{array}$ & $\begin{array}{c}\text { Surveillance } \\
\text { direction }\end{array}$ & $\begin{array}{c}\text { Surveillance } \\
\text { position }\end{array}$ & $\begin{array}{l}\text { Other } \\
\text { findings }\end{array}$ \\
\hline 1 & 2 & 118 & 210612 & 75 & 3 & 4 & 1 & U.b. * \\
\hline 2 & 2 & 118 & 210612 & 76 & 3 & 4 & 1 & U.b.* \\
\hline 3 & 2 & 118 & 210612 & 78 & 3 & 4 & 1 & U.b.* \\
\hline 4 & 2 & 118 & 210612 & 79 & 3 & 4 & 1 & U.b. * \\
\hline 5 & 2 & 118 & 210612 & 80 & 3 & 4 & 1 & U.b.* \\
\hline 6 & 2 & 118 & 210612 & 85 & 3 & 4 & 1 & U.b.* \\
\hline 7 & 2 & 118 & 210612 & 86 & 3 & 4 & 1 & U.b.* \\
\hline 8 & 2 & 118 & 210612 & 87 & 3 & 5 & 1 & U.b. * \\
\hline 9 & 2 & 118 & 210612 & 88 & 2 & 6 & 1 & U.b.* \\
\hline 10 & 2 & 118 & 210612 & 98 & 3 & 4 & 1 & U.b. * \\
\hline 11 & 2 & 118 & 210612 & 114 & 2 & 6 & 1 & U.b. ${ }^{*}$ \\
\hline 12 & 2 & 118 & 210612 & 118 & 2 & 4 & 1 & U.b.* \\
\hline 13 & 2 & 118 & 210612 & 120 & 1 & 7 & 1 & U.b.* \\
\hline 14 & 2 & 118 & 210612 & 121 & 1 & 5 & 1 & U.b. * \\
\hline 15 & 2 & 118 & 210612 & 124 & 2 & 8 & 1 & U.b. * \\
\hline
\end{tabular}

*Usnea barbata 
Table 6. $X X$ 2012. (PHE) Phenological phenomena monitoring (spring aspect)

\begin{tabular}{|c|c|c|c|c|c|c|}
\hline No. & $\begin{array}{c}\text { Sub-plot } \\
\text { no. }\end{array}$ & $\begin{array}{c}\text { Species } \\
\text { code }\end{array}$ & Event & $\begin{array}{c}\text { Observation } \\
\text { date }\end{array}$ & $\begin{array}{c}\text { Event } \\
\text { registered }\end{array}$ & Other findings \\
\hline 1 & 2 & 118 & 3 & 210612 & 1 & Usnea barbata \\
\hline 2 & 2 & 118 & 3 & 210612 & 1 & Usnea barbata \\
\hline 3 & 2 & 118 & 3 & 210612 & 7 & Usnea barbata \\
\hline 4 & 2 & 118 & 2 & 210612 & 7 & Usnea barbata \\
\hline 5 & 2 & 118 & 2 & 210612 & 7 & Usnea barbata \\
\hline 6 & 2 & 118 & 2 & 210612 & 7 & Usnea barbata \\
\hline 7 & 2 & 118 & 2 & 210612 & 7 & Usnea barbata \\
\hline 8 & 2 & 118 & 5 & 210612 & 7 & Usnea barbata \\
\hline 9 & 2 & 118 & 5 & 210612 & 7 & Usnea barbata \\
\hline 10 & 2 & 118 & 5 & 210612 & 7 & Usnea barbata \\
\hline 11 & 2 & 118 & 2 & 210612 & 7 & Usnea barbata \\
\hline 12 & 2 & 118 & 3 & 210612 & 7 & Usnea barbata \\
\hline 13 & 2 & 118 & 2 & 210612 & 7 & Usnea barbata \\
\hline 14 & 2 & 118 & 4 & 210612 & 1 & Usnea barbata \\
\hline 15 & 2 & 118 & 4 & 210612 & 1 & Usnea barbata \\
\hline
\end{tabular}

Table 7. XX 2012. (PHE) Phenological phenomena monitoring (autmn aspect)

\begin{tabular}{|c|c|c|c|c|c|c|}
\hline No. & $\begin{array}{c}\text { Sub-plot } \\
\text { no. }\end{array}$ & $\begin{array}{c}\text { Species } \\
\text { code }\end{array}$ & Event & $\begin{array}{c}\text { Observation } \\
\text { date }\end{array}$ & $\begin{array}{c}\text { Event } \\
\text { registered }\end{array}$ & Other findings \\
\hline 1 & 2 & 118 & 3 & 101012 & 1 & Usnea barbata \\
\hline 2 & 2 & 118 & 3 & 101012 & 1 & Usnea barbata \\
\hline 3 & 2 & 118 & 3 & 101012 & 7 & Usnea barbata \\
\hline 4 & 2 & 118 & 2 & 101012 & 7 & Usnea barbata \\
\hline 5 & 2 & 118 & 2 & 101012 & 7 & Usnea barbata \\
\hline 6 & 2 & 118 & 2 & 101012 & 7 & Usnea barbata \\
\hline 7 & 2 & 118 & 2 & 101012 & 7 & Usnea barbata \\
\hline 8 & 2 & 118 & 5 & 101012 & 7 & Usnea barbata \\
\hline 9 & 2 & 118 & 5 & 101012 & 7 & Usnea barbata \\
\hline 10 & 2 & 118 & 5 & 101012 & 7 & Usnea barbata \\
\hline 11 & 2 & 118 & 2 & 101012 & 7 & Usnea barbata \\
\hline 12 & 2 & 118 & 3 & 101012 & 7 & Usnea barbata \\
\hline 13 & 2 & 118 & 2 & 101012 & 7 & Usnea barbata \\
\hline 14 & 2 & 118 & 4 & 101012 & 1 & Usnea barbata \\
\hline 15 & 2 & 118 & 4 & 101012 & 1 & Usnea barbata \\
\hline
\end{tabular}

Table 8. XX 2012 (PHI) Phenological phenomena recording

\begin{tabular}{|c|c|c|c|c|c|c|c|}
\hline No. & $\begin{array}{c}\text { Sub-plot } \\
\text { no. }\end{array}$ & Tree no. & Event & $\begin{array}{c}\text { Observation } \\
\text { date }\end{array}$ & $\begin{array}{c}\text { Event } \\
\text { registered }\end{array}$ & $\begin{array}{c}\text { Surveillance } \\
\text { method applied }\end{array}$ & $\begin{array}{c}\text { Other } \\
\text { findings }\end{array}$ \\
\hline 1 & 2 & 75 & 3 & 210612 & 6 & 3 & Usnea barbata \\
\hline 2 & 2 & 76 & 3 & 210612 & 6 & 3 & Usnea barbata \\
\hline 3 & 2 & 78 & 3 & 210612 & 6 & 3 & Usnea barbata \\
\hline 4 & 2 & 79 & 2 & 210612 & 6 & 3 & Usnea barbata \\
\hline 5 & 2 & 80 & 2 & 210612 & 7 & 3 & Usnea barbata \\
\hline 6 & 2 & 85 & 2 & 210612 & 6 & 3 & Usnea barbata \\
\hline 7 & 2 & 86 & 2 & 210612 & 6 & 3 & Usnea barbata \\
\hline 8 & 2 & 87 & 5 & 210612 & 7 & 3 & Usnea barbata \\
\hline 9 & 2 & 88 & 5 & 210612 & 7 & 3 & Usnea barbata \\
\hline 10 & 2 & 98 & 5 & 210612 & 6 & 3 & Usnea barbata \\
\hline 11 & 2 & 114 & 2 & 210612 & 7 & 3 & Usnea barbata \\
\hline 12 & 2 & 118 & 3 & 210612 & 6 & 3 & Usnea barbata \\
\hline 13 & 2 & 120 & 2 & 210612 & 6 & 3 & Usnea barbata \\
\hline 14 & 2 & 121 & 4 & 210612 & 6 & 3 & Usnea barbata \\
\hline 15 & 2 & 124 & 4 & 210612 & 6 & 3 & Usnea barbata \\
\hline
\end{tabular}




\subsection{Litterfall Sampling and Analysis in 2012}

In $2012,1545.33 \mathrm{~kg} / \mathrm{ha}$ of litterfall ended up on the land surface of the forest ecosystem represented by the test plot. The litterfall was in the form of absolutely dry dead organic remnants produced by forest trees (assimilation organs of spruce and rowan trees, twigs, bark, blossoms, fruits etc.). The examined forest ecosystem deposited via litterfall to the land surface $77.48 \mathrm{~kg}$ of ashes and 1467.85 $\mathrm{kg}$ of combustible organic matter.

The most present of all nutrition macroelements in the litterfall collected was carbon, which comprised $47.23 \%$ of the litterfall. The total carbon inflow to the land via litterfall in the examined ecosystem amounted to $729.91 \mathrm{~kg} / \mathrm{ha}$.

Second most present nutrition element in the litterfall was nitrogen. A gram of litterfall contains $11.13 \mathrm{mg}$ of the total nitrogen. This means that $17.19 \mathrm{~kg}$ of the total amount of nitrogen reaches the land vial litterfall.

Table 9. XX2012 (LFP) Basic data on litterfall sampling

\begin{tabular}{|c|c|c|c|c|c|c|c|c|c|c|}
\hline \multirow[t]{2}{*}{ No. } & \multirow{2}{*}{$\begin{array}{l}\text { State } \\
\text { code }\end{array}$} & \multirow{2}{*}{$\begin{array}{c}\text { Sub- } \\
\text { plot } \\
\text { no. }\end{array}$} & \multirow[t]{2}{*}{ Latitude } & \multirow[t]{2}{*}{ Longitude } & \multirow[t]{2}{*}{ Altitude } & \multirow{2}{*}{$\begin{array}{l}\text { No. of } \\
\text { collectors }\end{array}$} & \multirow{2}{*}{$\begin{array}{l}\text { Total } \\
\text { collection } \\
\text { area }\end{array}$} & \multicolumn{2}{|c|}{$\begin{array}{l}\text { Sample collection } \\
\text { period }\end{array}$} & \multirow{2}{*}{$\begin{array}{l}\text { Other } \\
\text { findings }\end{array}$} \\
\hline & & & & & & & & from & to & \\
\hline 1 & 67 & 02 & $+43^{0} 17^{\prime} 30^{\prime \prime}$ & $+20^{\circ} 48^{\prime} 50^{\prime \prime}$ & $1712 / 35$ & 15 & 0.75 & 21.05 .12 & 19.10 .12 & \\
\hline
\end{tabular}

Table 10. XX2012 (LFM) Results of litterfall analysis

\begin{tabular}{|c|c|c|c|c|c|c|c|c|c|c|c|c|c|c|c|}
\hline \multirow{2}{*}{$\dot{z}$} & \multirow{2}{*}{ 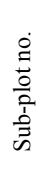 } & \multicolumn{2}{|c|}{ Collection period } & \multirow{2}{*}{$\begin{array}{l}\dot{0} \\
\stackrel{0}{0} \\
\text { Uू } \\
\stackrel{0}{0}\end{array}$} & \multirow{2}{*}{$\begin{array}{l}\tilde{0} \\
0 \\
\tilde{0} \\
\tilde{0} \\
\tilde{0} \\
\text { n. }\end{array}$} & \multirow{2}{*}{ 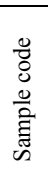 } & \multirow{2}{*}{ 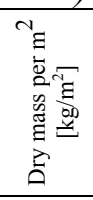 } & \multirow{2}{*}{ 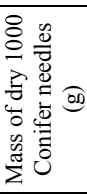 } & \multirow{2}{*}{$\begin{array}{l}\frac{80}{80} \\
\stackrel{0}{0} \\
\underset{0}{0}\end{array}$} & \multirow{2}{*}{ 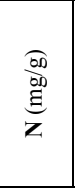 } & \multirow{2}{*}{$\begin{array}{l}\frac{b D}{b D} \\
\text { bD } \\
\text { a }\end{array}$} & \multirow{2}{*}{$\begin{array}{l}\frac{D D}{b D} \\
\text { bD } \\
\tilde{J} \\
\tilde{J}\end{array}$} & \multirow{2}{*}{ 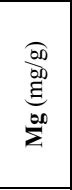 } & \multirow{2}{*}{$\begin{array}{l}\frac{\partial D}{b D} \\
\underline{B} \\
\simeq\end{array}$} & \multirow{2}{*}{ 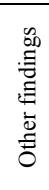 } \\
\hline & & from & to & & & & & & & & & & & & \\
\hline 1 & 2 & 21.05 .12 & 19.10 .12 & -9 & 118 & 11 & 0.1545 & 3.782 & 47.233 & 11.127 & 0.582 & 8.857 & 2.526 & 3.009 & \\
\hline
\end{tabular}

\subsection{Deposition Collection and Analysis}

At Level II test plots special attention is paid to wet deposition, whereby the most relevant is examination of the chemistry of the deposit in the immediate contact with plant organs where pollutants from the air remain (ICP Forests, 2010c).

In 2012 there were eight periods of collecting samples from collectors. 5 joint samples were collected from the "throughfall" collectors, 6 joint samples from "steamflow" collectors and 4 joint samples from "bulk" collectors. The results of the chemistry analyses of the samples are provided in Tables XX2012.PLD and XX2012.DEM. 


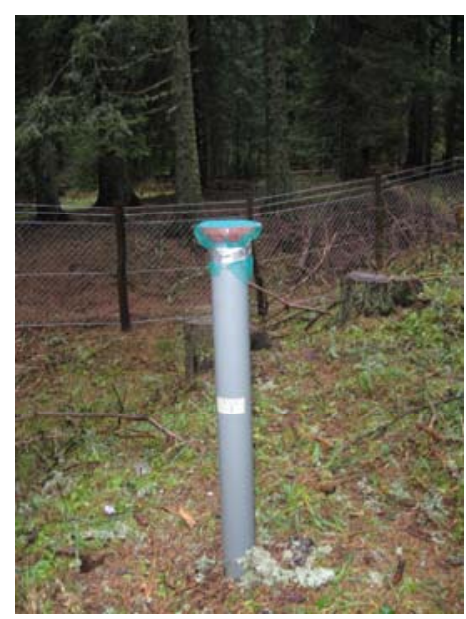

Figure 2. "Throughfall" collector

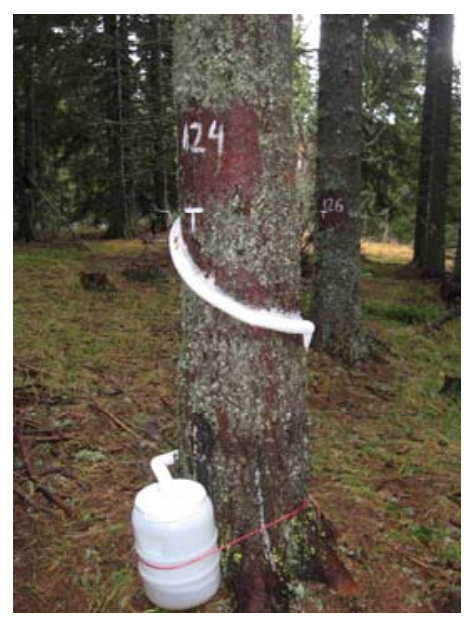

Figure 3. "Steamflow" collector

Table 11. XX2012(PLD) General data on the test plot for atmospheric deposition

\begin{tabular}{|c|c|c|c|c|c|c|c|c|c|c|c|c|c|c|}
\hline \multirow{2}{*}{$\dot{z}$} & \multirow{2}{*}{$\begin{array}{l}\text { 镸 } \\
\text { in }\end{array}$} & \multirow{2}{*}{ 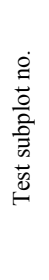 } & \multirow{2}{*}{ 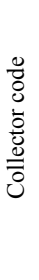 } & \multirow{2}{*}{$\begin{array}{l}\text { 芯 } \\
\text { 䔍 }\end{array}$} & \multirow{2}{*}{ 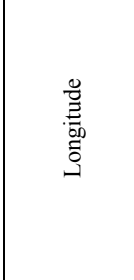 } & \multirow{2}{*}{ 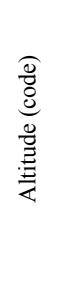 } & \multicolumn{2}{|c|}{$\begin{array}{l}\text { Active collection } \\
\text { period }\end{array}$} & \multirow{2}{*}{ 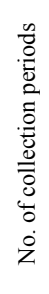 } & \multirow{2}{*}{$\begin{array}{l}\overline{\bar{z}} \\
\bar{\Xi} \\
\overline{0} \\
\stackrel{0}{0} \\
\overline{0}\end{array}$} & \multirow{2}{*}{ 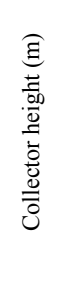 } & \multirow{2}{*}{ 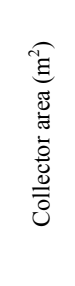 } & \multirow{2}{*}{ 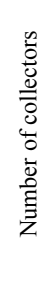 } & \multirow{2}{*}{ 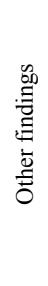 } \\
\hline & & & & & & & from & to & & & & & & \\
\hline 01 & 67 & 02 & 1 & $+43^{0} 17^{\prime} 30^{\prime \prime}$ & $+20^{\circ} 48^{\prime} 50^{\prime \prime}$ & 35 & 290212 & 230812 & 05 & 1 & 1.00 & 0.002 & 15 & \\
\hline 02 & 67 & 02 & 2 & $+43^{0} 17^{\prime} 30^{\prime \prime}$ & $+20^{\circ} 48^{\prime} 50^{\prime \prime}$ & 35 & 231211 & 190412 & 04 & 1 & 1.00 & 0.002 & 5 & \\
\hline 03 & 67 & 02 & 4 & $+43^{0} 17^{\prime} 30^{\prime \prime}$ & $+20^{\circ} 48^{\prime} 50^{\prime \prime}$ & 35 & 290212 & 230812 & 06 & 1 & 1.10 & 0.002 & 5 & \\
\hline
\end{tabular}

Table 12. XX2012(DEM) Laboratory analysis data for atmospheric deposition

\begin{tabular}{|c|c|c|c|c|c|c|c|c|c|c|c|c|c|c|c|c|c|c|}
\hline \multirow{2}{*}{ z } & \multirow{2}{*}{ 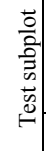 } & \multicolumn{2}{|c|}{$\begin{array}{l}\text { Collection } \\
\text { periods }\end{array}$} & \multirow{2}{*}{ 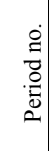 } & \multirow{2}{*}{ 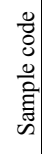 } & \multirow{2}{*}{ 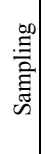 } & \multirow{2}{*}{$\stackrel{\mathbb{2}}{2}$} & \multirow{2}{*}{ 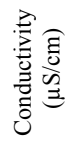 } & \multirow{2}{*}{ 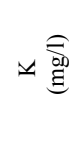 } & \multirow{2}{*}{ 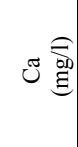 } & \multirow{2}{*}{ 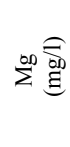 } & \multirow{2}{*}{ 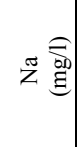 } & \multirow{2}{*}{ 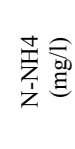 } & \multirow{2}{*}{ 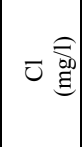 } & \multirow{2}{*}{$\begin{array}{l}\hat{O}_{z} \\
z_{z}\end{array}$} & \multirow{2}{*}{ 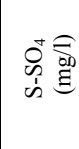 } & \multirow{2}{*}{ 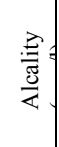 } & \multirow{2}{*}{ 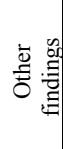 } \\
\hline & & from & to & & & & & & & & & & & & & & & \\
\hline 01 & 02 & 231211 & 180112 & 01 & 02 & 1 & 4.92 & 16.8 & 0.176 & 5.143 & .320 & 0.246 & 1.2 & 4.004 & 4.422 & 14.547 & 4.64 & \\
\hline 02 & 02 & 180112 & 290212 & 02 & 02 & 1 & 5.51 & 32.3 & 0.340 & 2.455 & 0.585 & 9.980 & 1.906 & 1.502 & 2.260 & 21.715 & 3.48 & \\
\hline 03 & 02 & 290212 & 150312 & 03 & 02 & 1 & 5.39 & 21.5 & 0.548 & 3.452 & 0.694 & 4.896 & 1.44 & 3.254 & 4.261 & 12.228 & 3.48 & \\
\hline 04 & 02 & 290212 & 150312 & 03 & 01 & 1 & 6.31 & 34.2 & 1.459 & 2.365 & 0.743 & 0.764 & 1.454 & 5.756 & 2.421 & 16.866 & 4.64 & \\
\hline 05 & 02 & 290212 & 150312 & 03 & 04 & 1 & 3.98 & 198.5 & 2.478 & 4.784 & 1.785 & 1.387 & 11.112 & 8.009 & 2.311 & 28.462 & 3.48 & \\
\hline 06 & 02 & 150312 & 190412 & 04 & 01 & 1 & 5.02 & 55.6 & 1.065 & 3.086 & 0.655 & 0.962 & 1.948 & 5.756 & 3.489 & 18.131 & 4.06 & \\
\hline 07 & 02 & 150312 & 190412 & 04 & 02 & 1 & 4.81 & 27.8 & 0.298 & 4.763 & 0.629 & 0.954 & 0.367 & 5.006 & 4.142 & 21.715 & 2.90 & \\
\hline 08 & 02 & 150312 & 190412 & 04 & 04 & 1 & 3.71 & 351 & 2.126 & 4.235 & 2.478 & 1.784 & 9.531 & 33.036 & 5.151 & \begin{tabular}{|l|}
43.009 \\
\end{tabular} & 1.74 & \\
\hline 09 & 02 & 190412 & 210512 & 05 & 01 & 1 & 5.78 & 21.7 & 1.147 & 1.220 & 0.383 & 0.670 & 0.191 & 4.004 & 7.761 & 30.781 & 4.64 & \\
\hline 10 & 02 & 190412 & 210512 & 05 & 04 & 1 & 4.17 & 123.6 & 2.315 & 3.438 & 1.233 & 16.19 & 3.692 & 8.009 & 11.134 & 29.938 & 1.74 & \\
\hline 11 & 02 & 210512 & 210612 & 06 & 01 & 1 & 6.3 & 31.1 & 3.876 & 8.953 & 0.974 & 1.268 & 0.777 & 4.505 & 6.040 & 25.089 & \begin{tabular}{|l|}
4.06 \\
\end{tabular} & \\
\hline 12 & 02 & 210512 & 210612 & 06 & 04 & 1 & 4.8 & 48.3 & 4.037 & 6.935 & 2.365 & 3.872 & 1.793 & 5.256 & 4.684 & 22.137 & 4.64 & \\
\hline 13 & 02 & 210612 & 190712 & 07 & 04 & 1 & 5.9 & 100.4 & 4.764 & 7.092 & 1.342 & 2.242 & 2.873 & 7.008 & 3.413 & 32.257 & 5.81 & \\
\hline 14 & 02 & 190712 & 230812 & 08 & 01 & 1 & 5.31 & 41.1 & 2.897 & 3.289 & 1.975 & 2.188 & 0.777 & 7.008 & 2.727 & 50.388 & 4.06 & \\
\hline 15 & 02 & 190712 & 230812 & 08 & 04 & 1 & 4.54 & 109.4 & 4.984 & 2.785 & 2.102 & 3.025 & 7.427 & 9.010 & 4.922 & 42.587 & 6.97 & \\
\hline
\end{tabular}




\subsection{Soil Solution Sampling and Analysis}

Tables 13. XX2012 (PSS) and 12. XX2014 (SSM) present the basic data on the soil solution measurements and chemical analyses of the collected samples of the soil solution.

Table 13. XX2012(PSS) basic data on the soil solution measurement

\begin{tabular}{|c|c|c|c|c|c|c|c|c|c|c|c|c|c|}
\hline$\dot{z}$ & 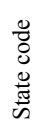 & 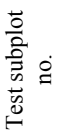 & 胥 & $\begin{array}{l}\stackrel{0}{\bar{E}} \\
\text { E. } \\
\bar{\Xi}\end{array}$ & 䒿 & $\begin{array}{l}\frac{\overrightarrow{0}}{0} \\
\stackrel{0}{0} \\
0\end{array}$ & 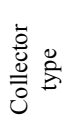 & 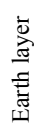 & 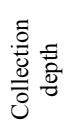 & 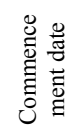 & 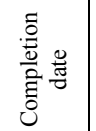 & 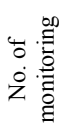 & 壹总 \\
\hline 01 & 67 & 2 & $+43^{0} 17^{\prime} 30^{\prime \prime}$ & $+20^{\circ} 48^{\prime} 50^{\prime \prime}$ & $1712 / 35$ & 1 & 2 & $\mathrm{H}$ & -0.30 & 271011 & 230812 & 03 & \\
\hline
\end{tabular}

Table 14. XX2012 (SSM) Data on the soil solution

\begin{tabular}{|c|c|c|c|c|c|c|c|c|c|c|c|c|c|c|c|c|c|}
\hline \multirow[t]{2}{*}{$\dot{z}$} & \multirow{2}{*}{ 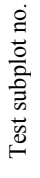 } & \multicolumn{2}{|c|}{ 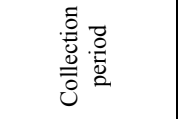 } & \multirow[t]{2}{*}{$\begin{array}{l}\dot{g} \\
\stackrel{\Xi}{0} \\
\stackrel{0}{\tilde{D}} \\
0\end{array}$} & \multirow[t]{2}{*}{$\begin{array}{l}\frac{\overrightarrow{0}}{0} \\
\frac{0}{0} \\
0\end{array}$} & \multirow[t]{2}{*}{ 胥 } & \multirow[t]{2}{*}{ 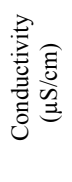 } & \multirow[t]{2}{*}{ 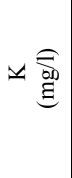 } & \multirow[t]{2}{*}{ 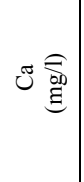 } & \multirow[t]{2}{*}{ 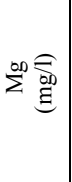 } & \multirow[t]{2}{*}{ 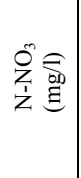 } & \multirow[t]{2}{*}{ 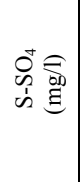 } & \multirow[t]{2}{*}{ 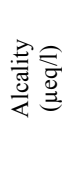 } & \multirow[t]{2}{*}{ 艺 $\widehat{\widehat{b j}}$} & \multirow[t]{2}{*}{$\begin{array}{l}\sum_{i}^{+} \\
\text {Z }\end{array}$} & \multirow[t]{2}{*}{ 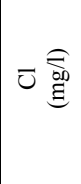 } & \multirow{2}{*}{ 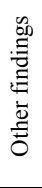 } \\
\hline & & from & to & & & & & & & & & & & & & & \\
\hline 01 & 02 & 271011 & 210512 & 01 & 00 & 4.8 & 341 & 5.984 & 10.654 & 3.243 & 30.032 & 30.781 & 2.32 & 5.438 & 10.392 & 14.015 & \\
\hline 02 & 02 & 210512 & 190712 & 02 & 00 & 6.92 & 122.5 & 6.953 & 12.558 & 2.428 & 24.404 & 24.245 & 2.90 & 11.190 & 2.231 & 5.006 & \\
\hline 03 & 02 & 190712 & 230812 & 03 & 00 & 6.21 & 88.0 & 9.876 & 9.475 & 3.254 & 15.618 & 28.673 & 4.06 & 5.945 & 2.019 & \begin{tabular}{|l|}
10.011 \\
\end{tabular} & \\
\hline
\end{tabular}

\subsection{Meteorological Monitoring}

According to the data obtained from the weather station Kopaonik of the Hydrometeorological Service of Serbia, mean monthly air temperatures exhibit regular annual patterns, with values on the rise from January to July and on decline toward the year-end. The coldest month in the period under review was February, with the mean monthly air temperature of $-6.9^{0} \mathrm{C}$, while the warmest month was July, when the mean monthly air temperature was $17.0^{\circ} \mathrm{C}$.

Mean monthly precipitation quantities ranged from $0.2 \mathrm{~mm}$ in August to $138.9 \mathrm{~mm}$ in January.

Mean monthly relative air humidity was the lowest in August and the highest in January. $\mathrm{ms}^{-1}$.

Mean monthly wind speed in the analyzed period ranged from 2.6 to 4.3

Table 15. Meteorological data for 2012 from weather station Kopaonik

\begin{tabular}{|c|c|c|c|c|c|c|c|c|c|}
\hline \multirow{2}{*}{ Month } & $\mathrm{PR}$ & $\mathrm{AT}$ & $\mathrm{AT} \min$ & $\mathrm{AT} \max$ & $\mathrm{RH}$ & $\mathrm{RH} \min$ & $\mathrm{RH} \max$ & WS & SR \\
\cline { 2 - 10 } & $\mathrm{mm}$ & ${ }^{\circ} \mathrm{C}$ & ${ }^{\circ} \mathrm{C}$ & ${ }^{\circ} \mathrm{C}$ & $\%$ & $\%$ & $\%$ & $\mathrm{~m} / \mathrm{s}$ & W.h/sq.m \\
\hline $\mathrm{I}$ & 138.9 & -6.5 & -9.5 & -3.5 & 95 & 90 & 99 & 3.6 & 468 \\
\hline II & 86.9 & -6.9 & -10.0 & -3.4 & 93 & 85 & 98 & 4.3 & 604 \\
\hline III & 47.6 & -0.4 & -4.4 & 4.5 & 76 & 54 & 91 & 3.0 & 1452 \\
\hline IV & 111.3 & 3.6 & -0.4 & 8.0 & 85 & 67 & 96 & 4.2 & 1592 \\
\hline V & 113.5 & 7.5 & 3.4 & 11.9 & 88 & 71 & 99 & 2.9 & 1436 \\
\hline VI & 47.8 & 14.6 & 8.9 & 19.7 & 72 & 49 & 93 & 3.0 & 2600 \\
\hline VII & 16.5 & 17.0 & 11.2 & 22.6 & 69 & 48 & 91 & 2.8 & 2397 \\
\hline VIII & 0.2 & 16.3 & 10.5 & 22.2 & 55 & 37 & 78 & 2.6 & 2305 \\
\hline IX & 22.5 & 13.2 & 8.2 & 18.4 & 66 & 48 & 85 & 3.2 & 1750 \\
\hline
\end{tabular}


Data obtained through research at Level II test plot Kopaonik were entered into the ICP on-line database in Hamburg.

\section{CONCLUSION}

As in the previous two years, parameters with continuous and an annualbasis frequency of monitoring were reviewed.

Crown assessment of the trees within Level II test plot Kopaonik was performed as at August 23, 2012.

As compared to the previous two years, the number of trees not subject to defoliation has increased, as well as the percentage of trees with weak defoliation, whereas the percentage of trees with moderate defoliation has declined. The percentage of trees with strong defoliation has remained almost the same as in the previous years.

The vegetation of the dominant species commenced rather late. Observed from different expositions of the evaluated trees, budding started in the third week of June 2012. Pollination lasted until mid-July.

In $2012,1545.33 \mathrm{~kg} / \mathrm{ha}$ of litterfall ended up on the land surface of the forest ecosystem represented by the test plot. The litterfall was in the form of absolutely dry dead organic remnants produced by forest trees. The examined forest ecosystem deposited via litterfall to the land surface $77.48 \mathrm{~kg}$ of ashes and $1467.85 \mathrm{~kg}$ of combustible organic matter. The most present of all nutrition macroelements in the litterfall collected was carbon, which comprised $47.23 \%$ of the litterfall. Second most present nutrition element in the litterfall was nitrogen. A gram of litterfall contains $11.13 \mathrm{mg}$ of the total nitrogen. This means that $17.19 \mathrm{~kg}$ of the total amount of nitrogen reaches the land vial litterfall.

In 2012 there were eight periods of collecting wet deposition samples from collectors and three periods of soil solution sampling.

The coldest month in the period under review was February with the mean monthly air temperature of $-6.9^{0} \mathrm{C}$, while the warmest month was July, when the mean monthly air temperature was $17.0^{\circ} \mathrm{C}$. Mean monthly precipitation quantities ranged from $0.2 \mathrm{~mm}$ in August to $138.9 \mathrm{~mm}$ in January.

\section{REFERENCES}

Nevenić, R., Rakonjac, LJ., Orlović, S. (2011): Praćenje uticaj zagađenja vazduha i njegovih efekata u šumskim ekosistemima na teritoriji Republike Srbije - monitoring stanja šuma Nivo I i Nivo II. Monografija. Institut za šumarstvo. Beograd. ISBN 978-86-8043928-0. UDK 630*1:502.175(497.11). pp. 1-294.

Monitoring and Impact Assessment of Air Pollution and its Effects in Forest Ecosystems on the Territory of the Republic of Serbia. Monograph; NFC Serbia - National Focal Center

***** (2010): MANUAL on methods and criteria for harmonized sampling, assessment, monitoring and analysis of the effects of air pollution on forests. United Nations Economic Commissions for Europe. Convention on Long-range Tran boundary Air Pollution. International Co-operative Programme on Assessment and Monitoring of Air Pollution 
Effects on Forests (ICP Forests). Programme Coordinating Centre of ICP Forests. Johann Heinrich von Thunen-Institute. Institute for World Forestry, Hamburg, Germany. ISBN 978-3926301-01-1. www.icp-forests.org/Manual.htm

***** (2010a): Manual on methods and criteria for harmonized sampling, assessment, monitoring and analysis of the effects of air pollution on forests - Parts I, II, IX, V, VII, VIII, IX, XVII; ISBN 978-3-926301-01-1, Edited in 2010

***** (2010b): Manual on methods and criteria for harmonized sampling, assessment, monitoring and analysis of the effects of air pollution on forests - Part X - Sampling and Analysis of Soil, ICP Forests, 2010, updated: 05/2010

***** (2010c): Manual on methods and criteria for harmonized sampling, assessment, monitoring and analysis of the effects of air pollution on forests - Part XIV - Sampling and Analysis of Deposition," ICP Forests, 2010, updated: 05/2010

***** (2010d): Europe`s Forests 1985-2010. 25 Years of Monitoring Forest Condition by ICP Forests. Hohann Heinrich von Thuunen - Institute, Institute for World Forestry. PCC of ICP Forests, Hamburg, Germany.

***** Forms and Explanatory Items To be applied for data submission 2011 onwards Version n7 Last update: 3 December 2012. http://www.icp-forests.org/page/datasubmission

Google 1 - http://www.icp-forests.org/Manual.htm

Google 2 - http://www.icp-forests.org/ 
